\title{
Behavior of Users Illustrated using Heavy-Tailed Distribution
}

\author{
Nripesh Trivedi \\ Indian Institute of Technology \\ (Banaras Hindu University) \\ Varanasi
}

\begin{abstract}
Behavior of users on online social services is characterized using heavy-tailed distribution. The assumption in heavytailed distribution is that few users are responsible for most of the variation. In this paper, users characterized using heavytailed distribution are further characterized according to their activity on the online social service. Further, this characterization of behavior is shown to have similar properties as a trigonometric function, $\sin x$.
\end{abstract}

\section{Keywords}

Heavy-tailed distribution, Users, Activity, Online social service

\section{INTRODUCTION}

Users participate in online social services [1] for different purposes. Some become part of these services to engage with other users while others become part of these services to engage with features of this online social service. In any case, it is important to understand behavior of users and categorize them accordingly. This paper categorizes the users into three categories based on their behavior (activity) and explains the properties of users using a simple trigonometric function.

\section{RELATED WORK}

Behavior of users is characterized in [1] using heavy-tailed distribution. In this paper, same characterization is further explained using user categorization and its properties are also explained. Behavior of users is also studied in [2], [3] and [4] using heavy-tailed distribution.

\section{METHODOLOGY}

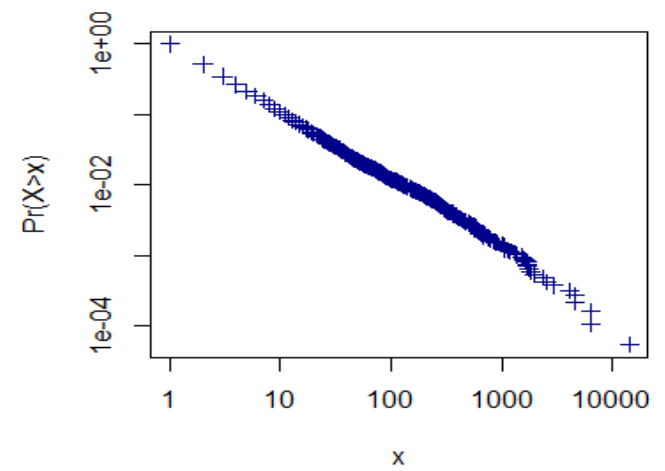

Figure 1: Behavior (Activity) of users represented using heavy-tailed distribution

Heavy-tailed distribution is a distribution that has a complementary cumulative distribution function which decreases at slower rate than decrease in exponential. It could be represented using the equation (1) below:

$$
\lim _{X \rightarrow \infty} e^{s X} R(X)=\infty
$$

The slower decrease in the right tail of heavy-tailed distribution causes lot of variation in $X$. From figure 1 , the users (along $x$-axis) could be categorized heuristically into three categories based on their activity on the online social service [1] (along $y$-axis). Users along $x$-axis could be heuristically divided into four quartiles based on their activity (along $y$-axis). The heuristic categorization using these four quartiles is as follows:

Users in the first quartile are irregular and infrequent users of the online social service due to their less activity on the online social service. That is, they rarely interact with other users or features of the online social service. It could be suitably assumed that this category of users suffer from some kind of depression, thus the lack of enthusiasm.

Users in the second and third quartile are regular and frequent users of the online social service due to their more activity on the online social service. That is, they are engaged with other users and features on the online social service. It could be suitably assumed that the life patterns of these users are influenced by the structure of online social service.

Users in the fourth quartile are the most regular and frequent users of the online social service as they are most active on the online social service. This category of users experiences the most engagement among all users. It could be suitably assumed that these users are alienated from other users as they are most influenced by the online social service.

In the three categories of the users, users are either more engaged with other users as in category 1 and category 2 or more engaged with the online social service as in category 3 . This behavior of users is periodic, that is, it is repeated after some interval of time. $\operatorname{Sin} x$ is a trigonometric function that is also periodic. It is shown in figure 2 below $\operatorname{Sin} x$ may be used to express almost any function. These functions could be found in real life situations just as $\sin x$ above.

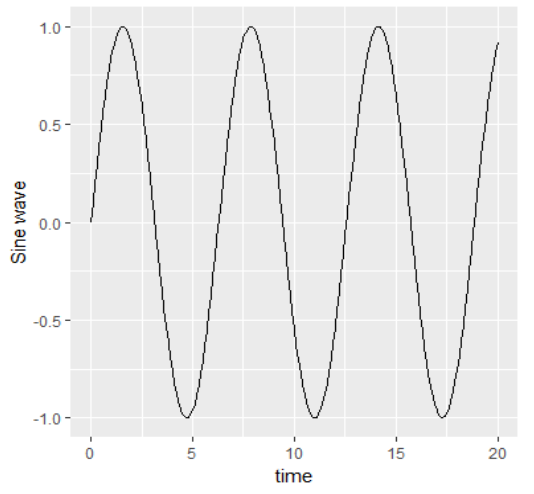

Figure 2: $\operatorname{Sin} x$ 


\section{CONCLUSION}

In this paper, behavior of users is studied using heavy-tailed distribution. Further, user behavior is found related to a trigonometric function. Further research on behavior of users includes finding relations between behavior of users and mathematical functions.

\section{REFERENCES}

[1] Trivedi, N., Asamoah, D. A., \& Doran, D. (2018). Keep the conversations going: engagement-based customer segmentation on online social service platforms. Information Systems Frontiers, 20(2), 239257.
[2] Viswanath, B., Mislove, A., Cha, M., \& Gummadi, K. P. (2009). On the Evolution of User Interaction in Facebook. Proceedings of the 2nd ACM Workshop on Online Social Networks - WOSN '09, 37.

[3] Anderson, A., Huttenlocher, D., \& Kleinberg, J. (2012). Discovering Value from Community Activity on Focused Question Answering Sites: A Case Study of Stack Overflow. Proceedings of the 18th ACM SIGKDD International Conference on Knowledge Dscovery and Data Mining, 850-858.

[4] Kwak, H., Lee, C., Park, H., \& Moon, S. (2010). What is Twitter, Social Network or a News Media? The International World Wide Web Conference Committee (IW3C2), 1-10. 